\title{
مبادئ التصميم الأمثلي للخزانات الخرسانية الأسطوانية المفتوحة

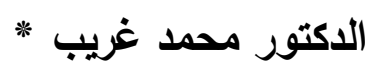

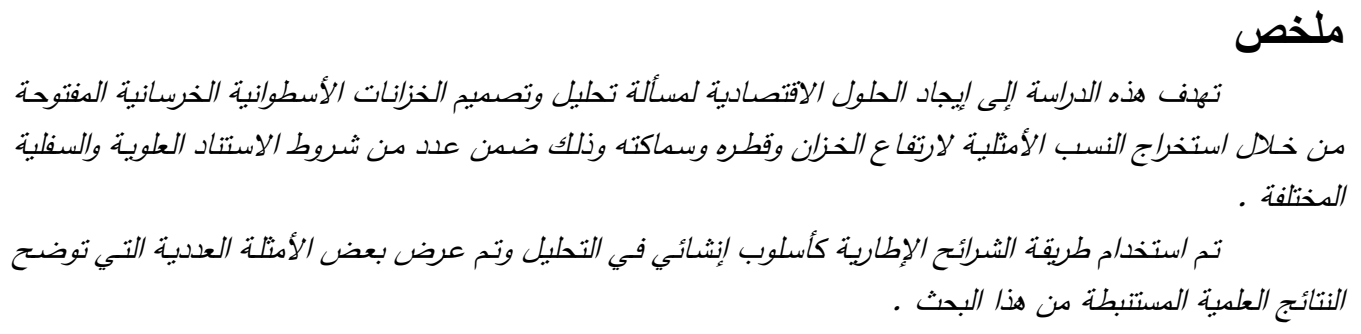

\begin{abstract}
The objective of this study is to find economic solutions for open cylindrical concrete tanks, in terms of different ratios of tank height, diameter and thickness, under several support conditions.

The equivalent strip method was utilized in the structural analysis procedures. Several numerical examples were presented, in order to clarify different aspects of this study.
\end{abstract}




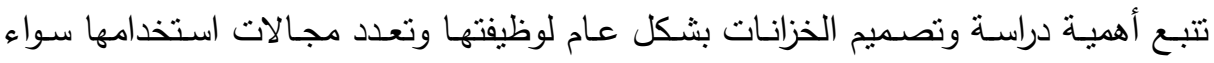

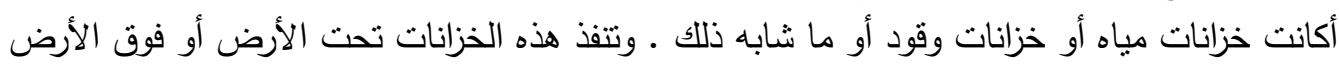

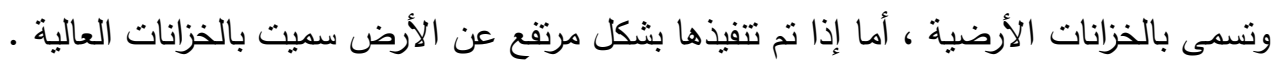

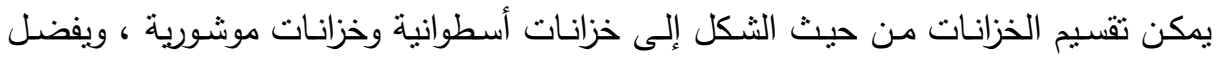

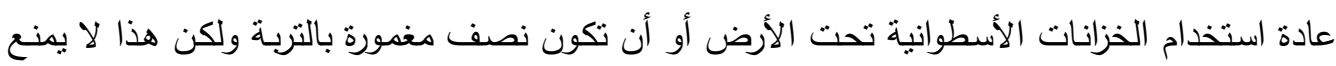

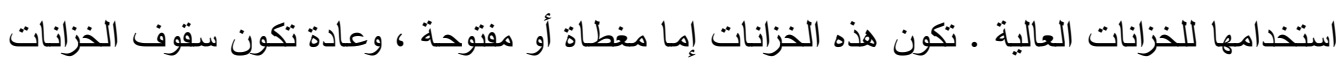

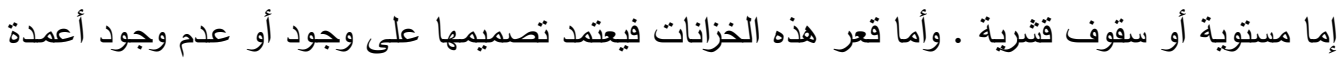

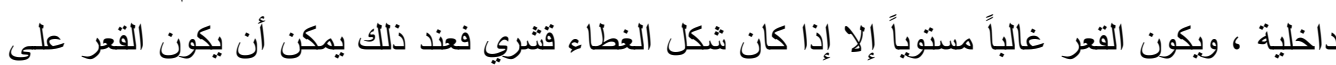

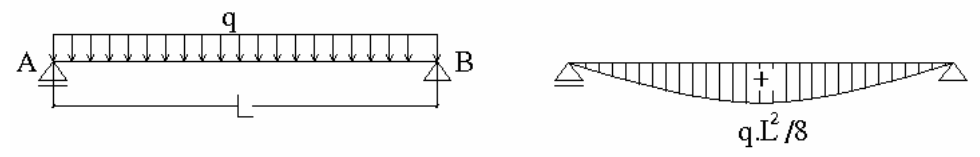

2.

نبين فيما يلي بعض الحالات الإنشائية المتسلسلة للوصول إلى فكرة البحث .

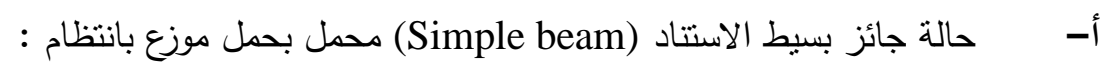

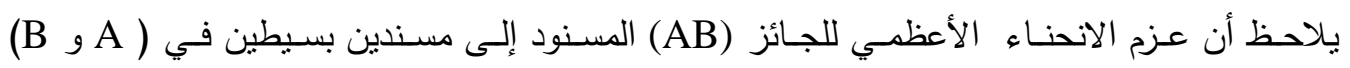
$0 M_{\max }=\frac{q \cdot L^{2}}{8}$ ومحمل بحمل موزع بانتظام شدنه (q) هوم الأناء ب- - حالة جائز موثوق الطرفين (Beam of fixed ends) ومحمل بحمل موزع بانتظام:

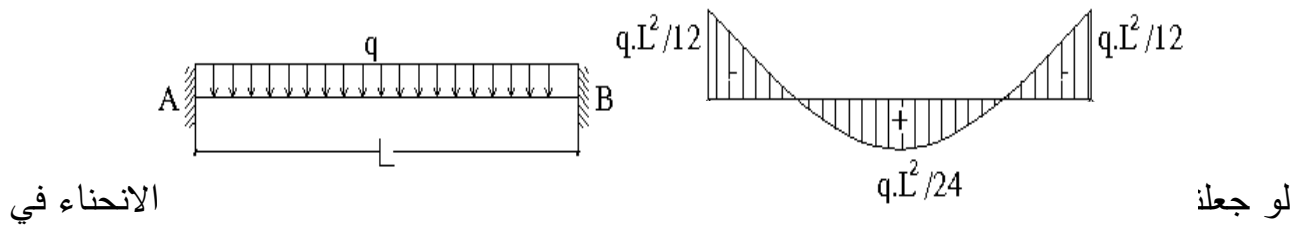


$M_{A}=M_{B}=-\frac{q \cdot L^{2}}{12}$ منتصف الجائز $M_{C}=+\frac{q \cdot L^{2}}{24}$ في حين أن عزم الانحناء في الوثاقة مما يدل على أن لنوعية المساند الطرفية : مساند مفصلية أو وثاقة تأثيراً كبيراً على قيم عزوم الانحناء .

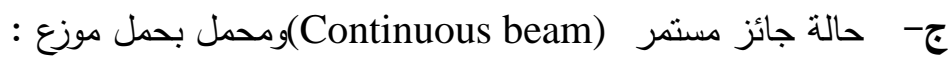
لو جعلنا الجائز البسيط (AB) المحمل بحمل موزع بانتظام شدته (q) وسطاً بين جائزين مماتلين له وبدون أي حمل عليهما لوجدنا أن عزم الانحناء في منتصف الجائز أصبح المسندين

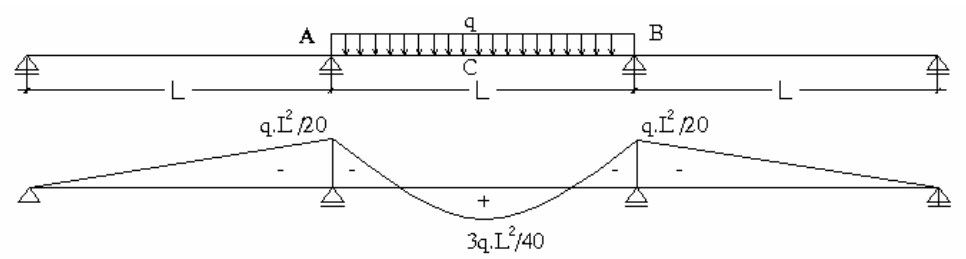

$$
M_{A}=M_{B}=-\frac{q \cdot L^{2}}{20}
$$

ومن أجل | $\left|M_{A}\right|=\left|M_{B}\right|=\frac{q \cdot L^{2}}{16}=\left|M_{C}\right|$

$$
\text { بطول قدره }
$$

وبشكل عام إذا كان الحمل الموزع بانتظام على كافة الجوائز شدته (q) كما في الثكل التالي :
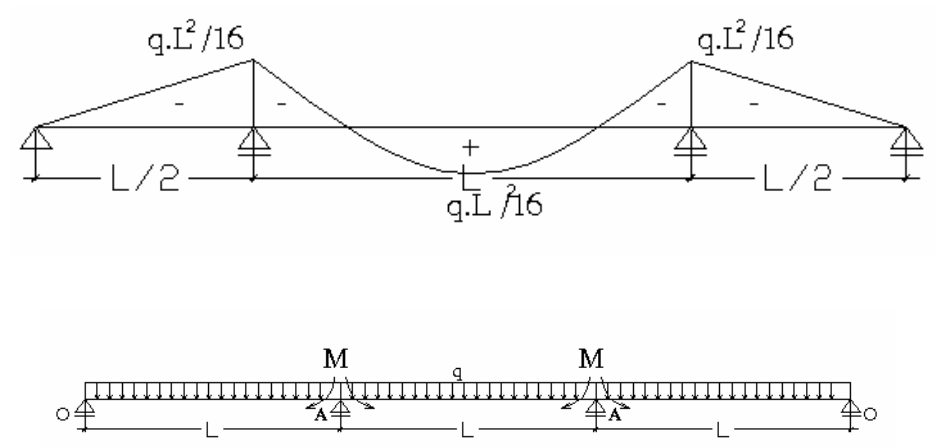
نجد أن قيم عزوم الانحناء (M) المستمر فوق المسندين الداخليين هي :

$$
\begin{aligned}
& 2 M(L+L)+M \cdot L=-2 \frac{q \cdot L^{3}}{4} \\
& \Rightarrow 3 M=-\frac{1}{4} q \cdot L^{2} \\
& M=-\frac{1}{12} q \cdot L^{2}
\end{aligned}
$$

ولحالة حمل موزع بانتظام على المجاز الوسطي وحمل متلثي التوزيع على المجازين الطرفيين نجد قيم عزوم الانحناء في هذه الحالة هي : بانئ $M=\frac{23 q . L^{2}}{300} \ldots, \ldots M=\frac{23 q . L^{2}}{300}$

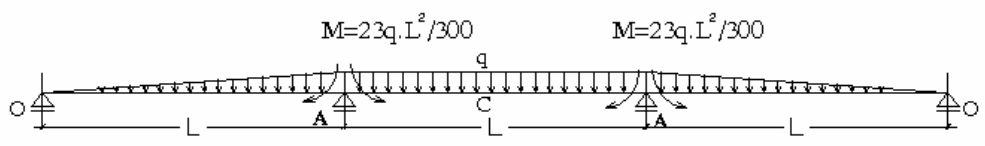




$$
\begin{aligned}
& 2 M_{A}(L+L)+M_{A} \cdot L=-\frac{8 q \cdot L^{3}}{60}-\frac{q \cdot L^{3}}{4}=\frac{23 q \cdot L^{3}}{60} \\
& 5 M_{A}=-\frac{23 q \cdot L^{2}}{60} \\
& \Rightarrow M_{A}=-\frac{23 q \cdot L^{2}}{300} \approx-\frac{q \cdot L^{2}}{13 \cdot 04} \\
& M_{C}=\frac{q \cdot L^{2}}{8}-\frac{23 q \cdot L^{2}}{300}=\frac{29 q \cdot L^{2}}{600} \approx \frac{q \cdot L^{2}}{20 \cdot 69}
\end{aligned}
$$

\section{3. الاراسة التحليلية للخزانات الأسطوانية (Cylindrical Tanks)}

تعد الخزانات الأسطوانية والخزانات المستطبلة من أهم الأشكال المستعملة في الخزانات المائية

• وقد سبقت دراسة الخزانات المستطيلة (3) ، وفيما يلي ستتم دراسة الخزانات الأسطوانية .

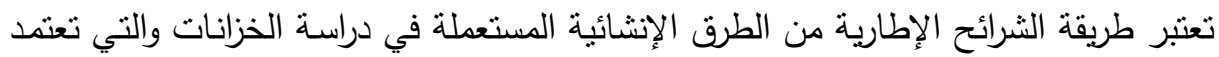

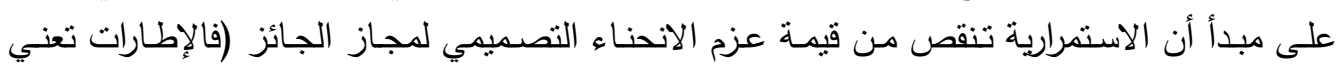

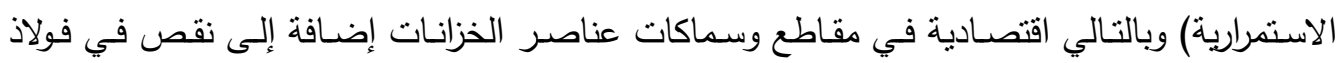

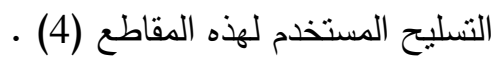
تتراوح نسبة حصة كل شريحة إطارية من الحمل الثاقولي بين 0.0-1.0 وذلك حسب النسبة الثبة (حيث H : ارتفاع جدار الخزان و D : قطر الخزان ) والتي تحدد قيمها بالمجال (0.5-2.0) كما D.

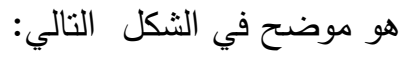




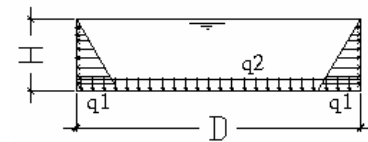

$$
\frac{\mathrm{H}}{\mathrm{D}}<0.5 \quad 0.5 \leqslant \frac{\mathrm{H}}{\mathrm{D}} \leqslant 2.0
$$

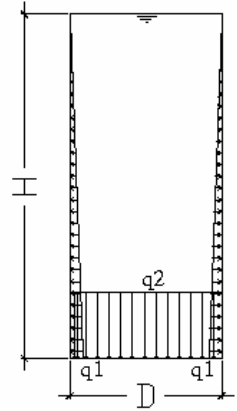

$2.0<\frac{\mathrm{H}}{\mathrm{D}}$

تم البحث بالحالة التي تكون فيها نسبة الحمل الذي يذهب بالعمل الإطاري في أحد الاتجاهين بالبلاطة مساوياً لنسبة الحمل الأفقي المطبق على الجدار في الإطار ذاته ، ويمكننا بيان ذلك وفقاً

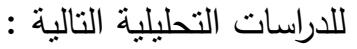
1-3 - 1الة تساوي السماكات

\section{' ـ حالة الاستناد الحر من الأعلى (جملة مقرة):}

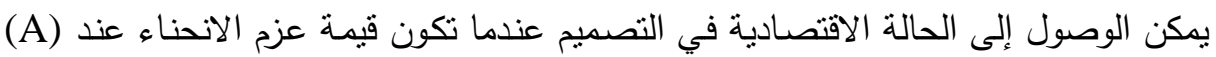

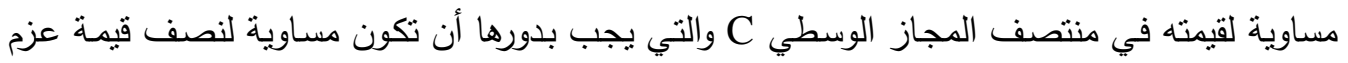

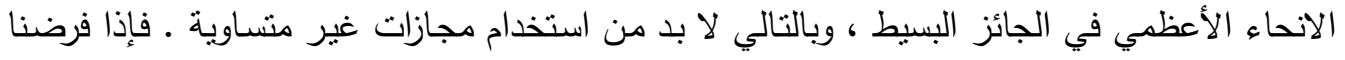

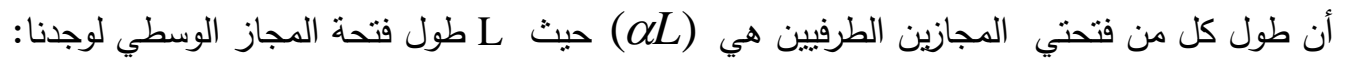

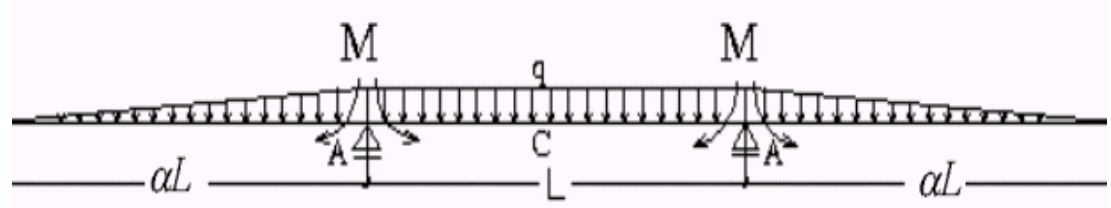




$$
\begin{aligned}
& M_{C}=\frac{q L^{2}}{8}-\frac{q \cdot(\alpha L)^{2}}{6}=\frac{q \cdot L^{2}}{16} \\
& \frac{1}{8}-\frac{\alpha^{2}}{6}=\frac{1}{16} \Rightarrow \alpha^{2}=\frac{6}{16} \\
& \alpha=\frac{\sqrt{6}}{4}=0.612
\end{aligned}
$$

إذاً يكون الخزان اقتصادياً من أجل ارتفاع لجدرانه يساوي 0.612 من قطره ، وذلك لحالة الاستناد

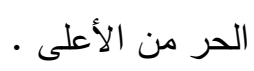

بـ حالة الاستناد البسيط من الأعلى (جملة غير مقرة):

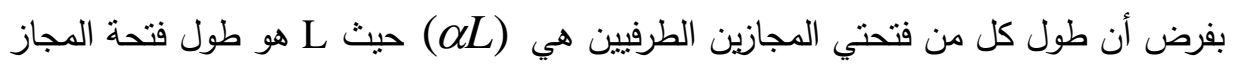

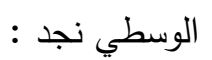

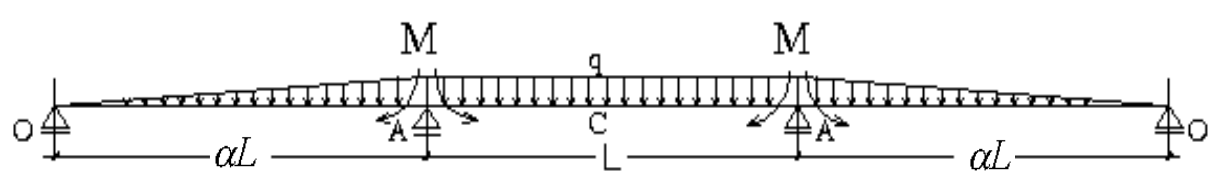

$$
\begin{aligned}
& 2 M_{A}(\alpha L+L)+M_{A} \cdot L=-\frac{8 q(\alpha L)^{3}}{60}-\frac{q \cdot L^{3}}{4}=-\frac{q \cdot L^{3}}{60}\left(8 \alpha^{3}+15\right) \\
& M_{A}(2 \alpha+3)=-\frac{q \cdot L^{2}}{60}\left(8 \alpha^{3}+15\right) \\
& \Rightarrow M_{A}=-\frac{q \cdot L^{2}}{60} \cdot \frac{8 \alpha^{3}+15}{2 \alpha+3}
\end{aligned}
$$

$$
\begin{aligned}
& \text { ومن أجل } \frac{q \cdot L^{2}}{16}=\left|M_{A}\right|=\left|M_{C}\right| \text { عندها تكون } \frac{q \cdot L^{2}}{60} \frac{8 \alpha^{3}+15}{2 \alpha+3}=\frac{q \cdot L^{2}}{16} \\
& \Rightarrow \frac{8 \alpha^{3}+15}{2 \alpha+3}=3.75 \Rightarrow 8 \alpha^{3}+15=7.5 \alpha+11.25 \\
& 8 \alpha^{3}-7.5 \alpha+3.75=0
\end{aligned}
$$


نستطيع بالحصول على قيمة م متحديد أبعاد الخزان الاقتصادي المراد تصميمه وذللك باختيار

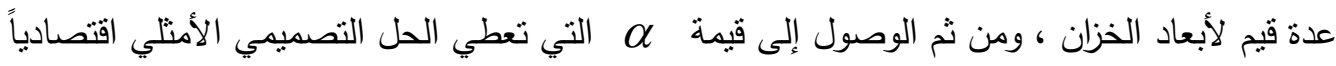

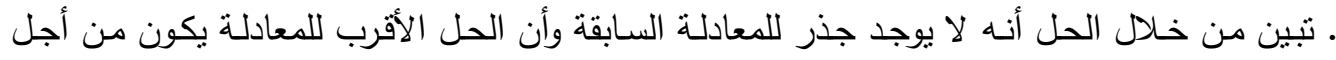

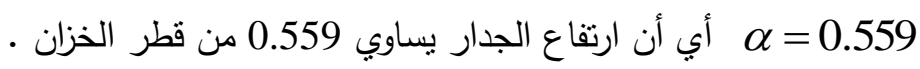

2-3-3 حالة عدم تساوي السماكات (ما بين قعر الخزان وجداره)

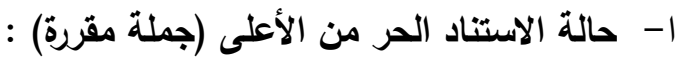
لا يوجد تعديل على النتيجة السابقة والتي يكون الخزان اقتصادياً من أجل ارتفاع لجاد لجداره يساوي من قطره . ب- - حالة الاستتاد البسيط من الأعلى (جملة غير مقررة).

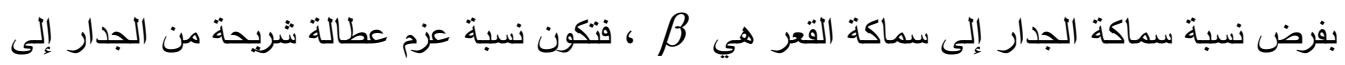

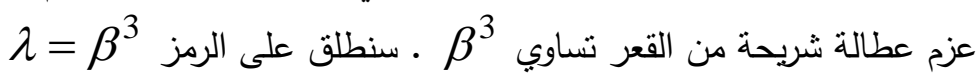
عندها تصبح المعادلات كالتالي:

$$
\begin{aligned}
& 2 M_{A}\left(\frac{\alpha L}{\lambda \cdot I}+\frac{L}{I}\right)+M_{A} \cdot \frac{L}{I}=-\frac{8 q \cdot L^{3}}{60 \lambda \cdot I}-\frac{q \cdot L^{3}}{4 I} \\
& M_{A}\left(\frac{2 \lambda}{3}+3\right)=-\frac{q \cdot L^{2}}{60}\left(\frac{8 \alpha}{\lambda}+15\right) \\
& \Rightarrow M_{A}=-\frac{q \cdot L^{2}}{60}\left(\frac{8 \alpha}{\lambda}+15\right) /\left(\frac{2 \lambda}{3}+3\right) \\
& M_{C}=\frac{q \cdot L}{2} \cdot \frac{L}{2}-\frac{q}{2} \frac{L^{2}}{4}-M_{A} \\
& M_{C}=M M_{A} \\
& 2 M_{A}=q \frac{L^{2}}{8} \Rightarrow M_{A}=q \frac{L^{2}}{16} \\
& q \frac{L^{2}}{16}=-\frac{q \cdot L^{2}}{60}\left(\frac{8 \alpha}{\lambda}+15\right) /\left(\frac{2 \lambda}{3}+3\right)
\end{aligned}
$$




$$
\begin{aligned}
& -\left(\frac{2 \lambda}{3}+3\right)(3.75)+8 \frac{8 \alpha^{3}}{\lambda}+15=0 \\
& 8 \frac{\alpha^{3}}{\lambda}-7.5 \frac{\alpha}{\lambda}+3.75=0
\end{aligned}
$$

من المعادلة الأخيرة ، يمكن استتناج مخطط بياني يربط نسبة ارتفاع الجدار إلى قطر القاعدة ، مع نسبة سماكتي الجدار والقاعدة كما في الثكل (1) التالي :

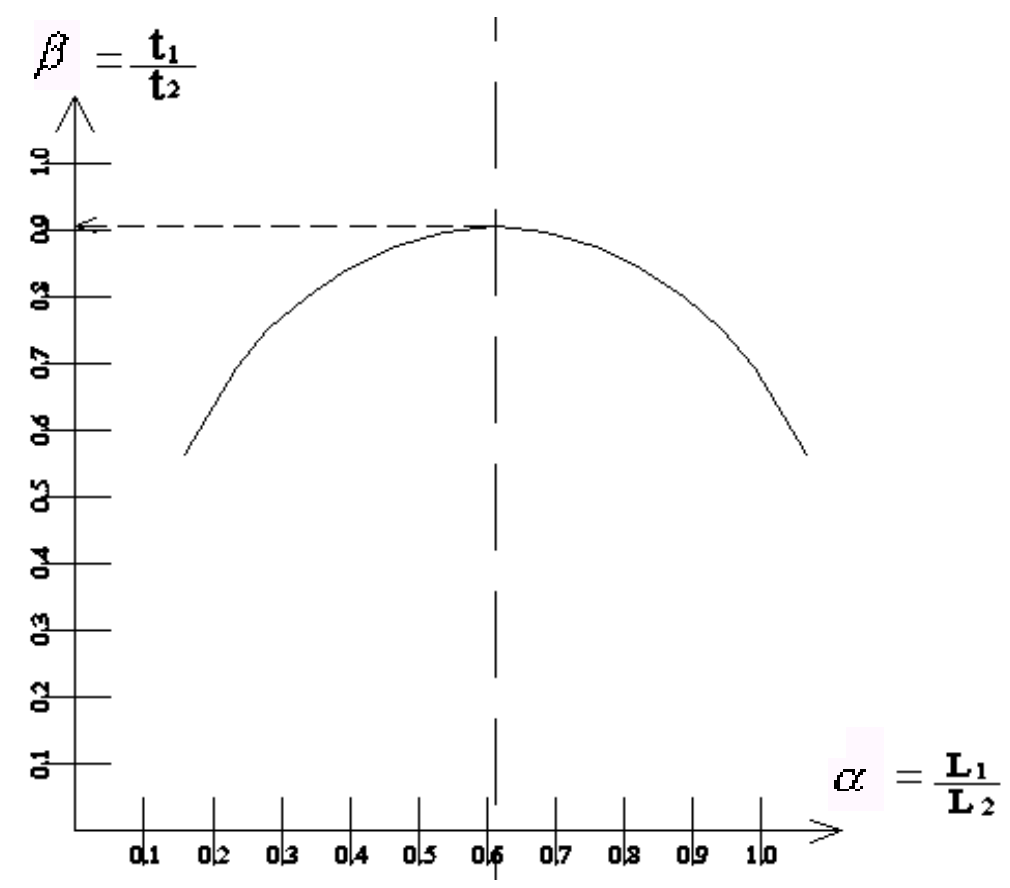

- يدل الخط المتقطع إلى حالة الاستناد الحر من الأعلى ، بينما يدل الخط المستمر إلى حالة الاستناد البسيط من الأعلى -

الشكل (1) مخطط بياني يريط نسبة ارتفاع الجدار إلى قطر القاعدة مع نسبة سماكة الجدار إلى سماكة القاعدة الجأ 
بملاحظة الثكل السابق ند أنه من أجل فرق في الاختيار الاقتصادي بين الجملة المقرة وغير المقررة (طبيعة استتاد جدار الخزان من الأعلى

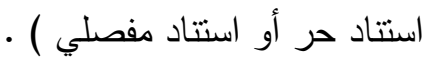

\section{4. مناقثة عامة لتصميم أمثلي لخزان أسطواني ذو سعة محددة}

المطلوب دراسة أمثلية أولية لأبعاد وتسليح خزان أسطواني يتسع لحجم V(m²

$$
\begin{aligned}
& \text { الحجمي ر (6,5). } \\
& \text { خطوات الحل : } \\
& \text { 1- يعطى حجم الخزان الأسطواني بالعلاقة: } \\
& V=\pi \frac{L^{2}}{4} \cdot \alpha L \\
& \Rightarrow L=\sqrt[3]{\frac{4 . V}{\pi \cdot \alpha}}
\end{aligned}
$$

2- بفرض قيمة للعامل م ، يمكن إيجاد قطر الخزان L من العلاقة السابقة ، وكذلك ارتفاع جدران

$$
\text { الخزان }
$$

3- تحديد نسبة سماكة جدار الخزان إلى سماكة بلاطة القعر ، من خلال الشكل (1) المستخرج في

$$
\text { البحث. }
$$

4- تحديد عزم الانحناء الأعظمي في البلاطة والجدار من خلال العلاق التالية:

$$
M_{A}=q \frac{L^{2}}{16}
$$

ومن ثم اختيار السماكة الدنيا لمقطع (جدار الخزان) من خلال علاقات تصميم مقطع خرساني معرض للانحناء ويشترط فيه الكتامة المائية .

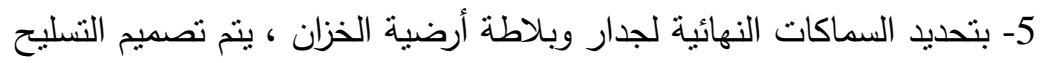
المطلوب لكل من الجدار وأرضية الخزان. 
6-مقارنة الحلول الأولية المتاحة ، واختيار الحل الاقتصادي النهائي من خلال حجم الخرسانة ووزن

النسليح الناتج.

مثال عددي

المطلوب تصميم أمثلي لخزان مياه أسطواني خرساني مفتوح من الأعلى والجدار مستتد استتاداً بسيطاً

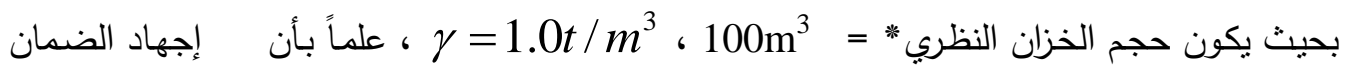

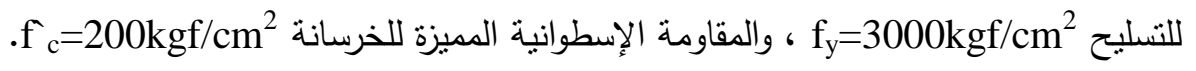

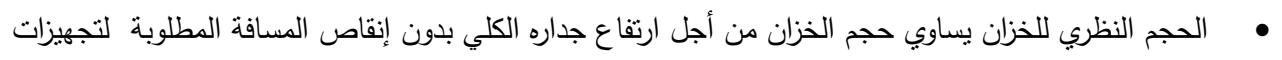

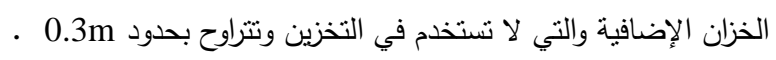

$$
\begin{aligned}
& \text { الحل }
\end{aligned}
$$

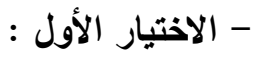

$$
\begin{aligned}
& \text { 1- يعطى حجم الخزان الأسطواني بالعلاقة : } \\
& V=\pi \frac{L^{2}}{4} . \alpha L \\
& \Rightarrow L=\sqrt[3]{\frac{400}{\pi \cdot \alpha}}
\end{aligned}
$$

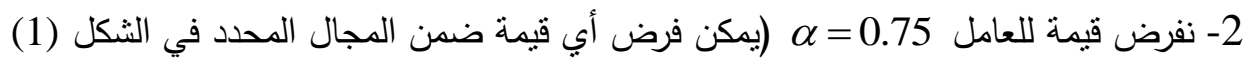
وبالتالي يعطى قطر الخزان L بالقيمة:

$$
\begin{aligned}
& L=\sqrt[3]{\frac{400}{\pi \times 0.75}}=5.537 m \cong 5.55 m \\
& H=\alpha . L=0.75 \times 5.55=4.16 \cong 4.15 m \text { ارتفاع جدار الخزان }
\end{aligned}
$$

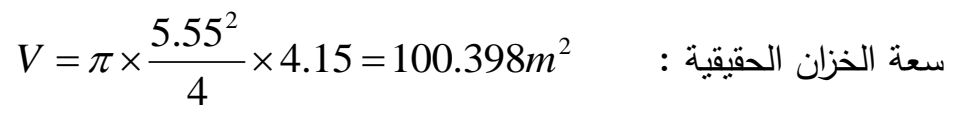

3- تؤخذ نسبة سماكة جدران الخزان إلى سماكة بلاطة الأرضية $0.875=$ 


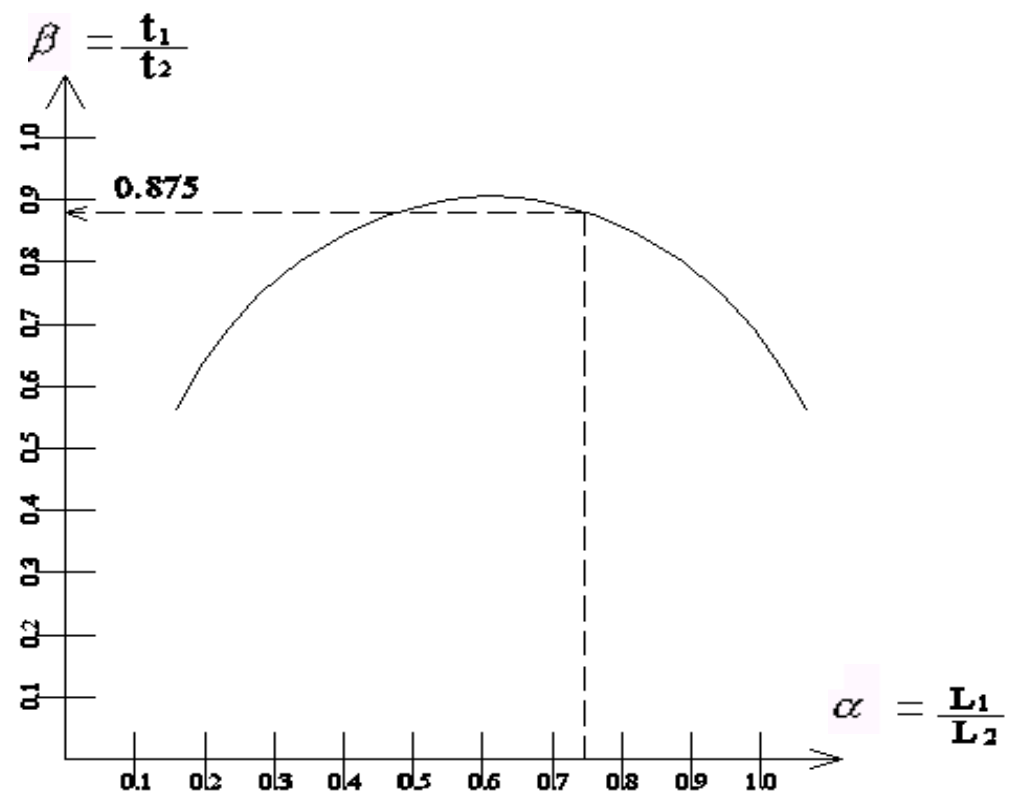

4- يعطى العزم الأعظمي في بلاطة الأرضية والجدار من العلاقة التالية :

$$
M=\frac{q . l^{2}}{16}=\frac{4.15 \times 5.55^{2}}{16}=7.99 \mathrm{t} . \mathrm{m}
$$

تحدد السماكة الدنيا لجدار الخزان من خلال علاقات تصميم مقطع خرساني معرض للانحناء :

$$
\sigma_{c}=0.3 \times 200=60 \mathrm{~kg} / \mathrm{cm}^{2}, \sigma_{s}=0.55 \times 3000=1650 \mathrm{kgf} / \mathrm{cm}^{2}
$$

$\gamma_{o}=n \frac{\sigma_{c}}{\sigma_{s}}=15 \frac{60}{1650}=0.545 \Rightarrow \gamma_{h}=2.54$

$$
d=\gamma_{h} \sqrt{\frac{M}{\sigma_{c} \cdot b}}=2.54 \sqrt{\frac{7.99 \times 10^{5}}{60 \times 100}}=29.3
$$

سماكة الجدار من شرط الكتامة المائية لمقطع معرض لعزم انحناء كما يلي (7) :

$$
t \geq \sqrt{\frac{M}{3}}=\sqrt{\frac{7990}{3}}=51.6 \mathrm{~cm}
$$




$$
\text { t سماكة جدار الخزان the }
$$

وبالتالي تكون سماكة بلاطة أرضية الخزان

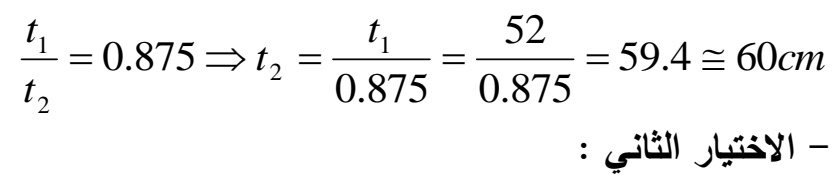

$$
\text { لنفرض قيمة المعامل } 12 \alpha=0.6
$$

\begin{tabular}{|c|c|c|}
\hline $\begin{array}{c}\text { السماكة الدنيا } \\
\text { cm }\end{array}$ & $\begin{array}{c}\text { القطر أو الطول } \\
\text { m }\end{array}$ & العنصر \\
\hline 57 & 5.95 & قعر الخزان \\
\hline 52 & 3.65 & جدار الخزان \\
\hline
\end{tabular}

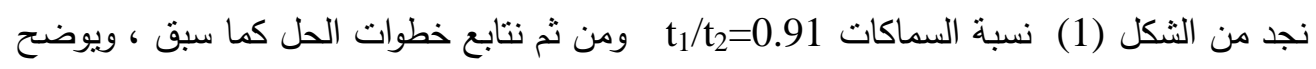
الجدول التالي النتائج النهائية للاختيار الثاني :

ملاحظات :

- - تم الاكتفاء بتصميم الأبعاد الخرسانية ضمن هذه الورقة .

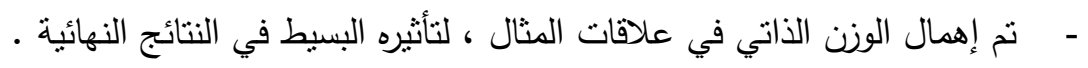

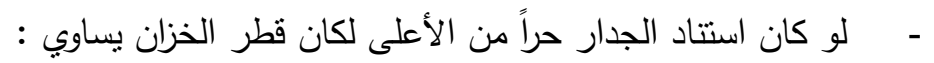

$$
L=\sqrt[3]{\frac{400}{\pi \times 0.0 .612}}=5.93 \mathrm{~m}
$$

\begin{tabular}{|c|c|c|}
\hline $\begin{array}{c}\text { السماكة الدنيا } \\
\text { cm }\end{array}$ & القطر أو الطول & العنصر \\
\hline 52 & 5.95 & قعر الخزان \\
\hline 52 & 3.65 & جدار الخزان \\
\hline
\end{tabular}

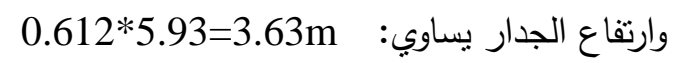

وتنتخرج سماكة القعر والجدار من خلال العلاقات التصميمية ـ ويوضح الجدول النالي النتائج النهائية

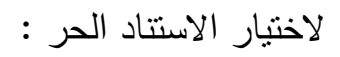




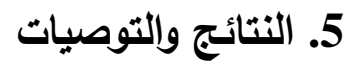

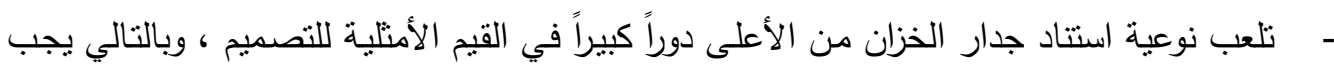

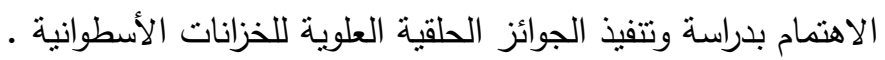

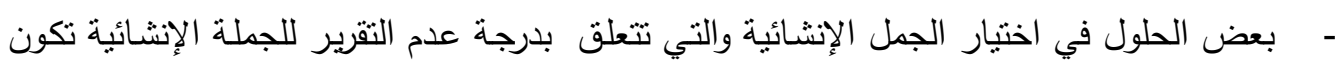

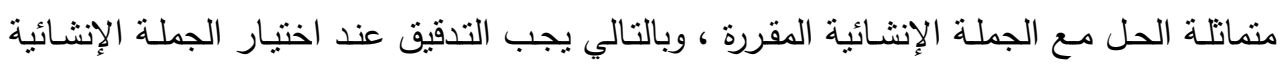
الفعالة .

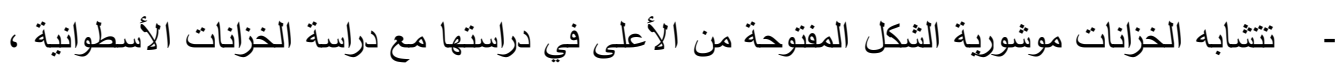

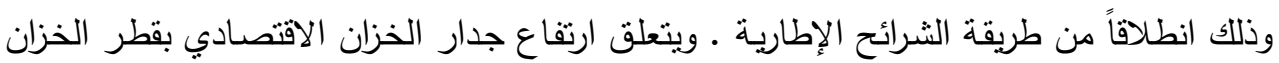

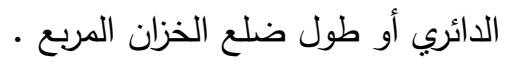
- إن الطريقة المنبعة في البحث هي طريقة بسيطة وسريعة في تصميم الخزانات الأسطوانية لتحديد

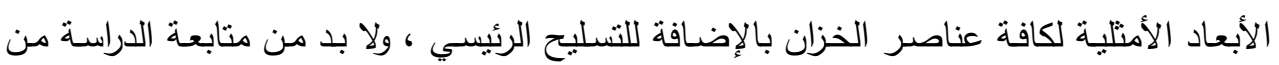

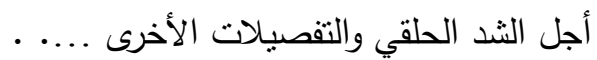

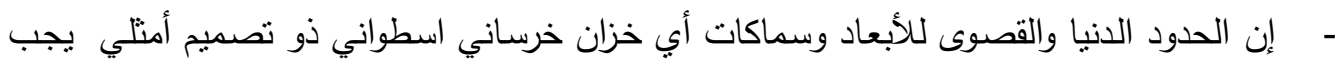
أن تكون محصورة بين القيم التالية :

$$
\begin{aligned}
& \frac{L_{1}}{L_{2}} \equiv 0.20 \rightarrow 1.0 \quad \text { ، } \quad \frac{t_{1}}{t_{2}} \cong 0.55 \rightarrow 0.91 \\
& \text { حيث: th : سماكة الجدران : t : سماكة قعر الخزان }
\end{aligned}
$$

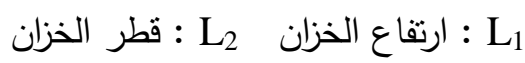


[1] تـادفي ، سـليمان محمد، خزانـات الميـاه العاليـة والأرضـية، دار الغدير ســية الطبعـة الثانيـة ،

01992

[2] Bakhoum, Michel Structural Mechanics, Volume (1), Cairo, Egypt, 1992

[3] هولا ، إبراهيم _غريب ، محمد _ علوني ، غيات دراسـة الخزانات منوازية المسنطيلات، مجلة باسل

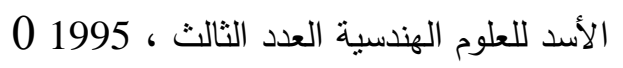

[4] Gray and Manning, Concrete Water Towers. , 1989.

[5] Roland "Structural Design in Reinforced Concrete", U.S.A, 1993.

[6] Southland and Reese ,Reinforced Concrete Design, John Wiley, 1992.

[7] Hilal , M. "Theory and Design of Reinforced Concrete Tanks" ,Published by J MARCOU. \& Co. 24, Abdel Khalek Sarwat Street- Cairo, 1976. 